Evaluar.

2004, $n^{\circ} 4$ (setiembre)
Laboratorio de Evaluación Psicológica y Educativa. Facultad de Psicología Universidad Nacional de Córdoba (Argentina). ISSN N ${ }^{\circ} 1515$ - 1867

\title{
Una perspectiva Social Cognitiva de la transición entre la escuela y el trabajo
}

\author{
Robert W. Lent*1 ${ }^{1}$, Gail Hackett** \& Steven D. Brown*** \\ Traducción: Dr. Edgardo Pérez \& Lic. Fabián Olaz
}

* Robert W. Lent es profesor en el Department of Counseling and Personnel Services, University of Maryland, College Park.

**Gail Hackett es profesor en la Division of Psychology in Education, Arizona State University, Tempe. ***Steven D. Brown es profesor en el Department of Counseling Psychology, Loyola University Chicago, Wilmette, IL.

Resumen: Este artículo propone a la Teoría Social Cognitiva del Desarrollo de Carrera (SCCT; Lent, Brown, \& Hackett, 1994) como una ventajosa perspectiva para comprender el proceso de transición entre la escuela y el trabajo. En lugar de enfatizar solo el período inmediatamente precedente al egreso de la escuela media, la perspectiva SCCT del proceso de transición entre la escuela y el trabajo se concentra en seis aspectos relacionados evolutivamente que se manifiestan a través de los años escolares. El énfasis en estos seis temas (formación de autoeficacia y expectativas de resultados, desarrollo de los intereses, relaciones entre intereses y metas, traducción de metas en acciones, ejecución de destrezas ; negociación de apoyos y obstáculos de transición) sugiere múltiples metas e intervenciones tendientes a facilitar el desarrollo de carrera de los estudiantes durante los años de escuela y después del ingreso al trabajo.

Palabras Clave: Teoría Social Cognitiva de la Carrera- Transición Escuela-Trabajo

\section{Una perspectiva social cognitiva de la transición entre la escuela y el trabajo}

Hace pocos años presentamos una teoría social cognitiva del desarrollo de carrera (SCCT) relacionada con el proceso mediante el cual las personas forman sus intereses académicos y ocupacionales, realizan elecciones en esos ámbitos y alcanzan diferentes

\footnotetext{
1 La correspondencia relacionada con este artículo debe ser dirigida a Robert W. Lent, Department of Counseling and Personnel Services, College of Education, University of Maryland, College Park, MD 20742.E-mail: RL95@umail.umd.edu.
} 
niveles de éxito en la escuela o el trabajo (Lent, Brown, \& Hackett, 1994). Basado en la teoría social cognitiva de Bandura (1986), este marco conceptual enfatiza varias variables de agencia personal (autoeficacia, expectativas de resultados, metas) - y la interacción de las mismas con otras importantes variables personales y ambientales (ej.., genero, raza, apoyos y obstáculos sociales) - en el contexto del desarrollo de carrera de las personas. SCCT ha recibido mucha atención de los investigadores, incluyendo algunos intentos recientes de verificar varias de sus proposiciones en minorías raciales, estudiantes universitarios y pre-universitarios (Fouad \& Smith, 1996; Gainor \& Lent, in press; Lapan, Shaughnessy, \& Boggs, 1996; Lopez, Lent, Brown, \& Gore, 1997).

Creemos que el análisis SCCT de los intereses vocacionales y educacionales, la elección y el rendimiento suministra una perspectiva útil para comprender el desarrollo de atributos básicos y competencias de carrera durante la escuela y después de la misma. El enfoque SCCT también parece tener consecuencias prácticas para todas las tentativas de promover el desarrollo de carrera en un amplio espectro de orientados (Betz \& Hackett, 1997; Brown \& Lent, 1996; Chartrand \& Rose, 1996; Hackett \& Byars, 1996). Aunque la teoría no fue específicamente diseñada para explicar el proceso de transición escuela-trabajo, existen posibilidades de extender este marco conceptual para comprender $y$, quizá, facilitar esta transición del desarrollo (Lent, Hackett, \& Brown, 1996). En este artículo, sugerimos varias direcciones para esa ampliación de la teoría.

En particular, después de abordar brevemente el contexto actual de la transición escuela-trabajo y las reformas emprendidas, desarrollaremos seis temas vinculados del desarrollo y derivados de la teoría SCCT, que pueden ayudar a comprender el proceso de transición hacia el trabajo.

\section{El contexto de las reformas para la transición escuela-trabajo}

Una variedad de iniciativas locales y federales han surgido recientemente como respuesta a percepciones ampliamente compartidas respecto a que las escuelas no están preparando adecuadamente a los estudiantes para transitar desde los ámbitos escolares a los ocupacionales. Mientras que esas propuestas han estado dirigidas a asistir a todos los estudiantes, ha habido una preocupación particular relacionada con el ajuste al trabajo de un gran número de estudiantes que debido a determinadas circunstancias se 
ven obligados a trabajar sin completar su educación superior (Olson, 1997b). Algunas de las iniciativas más populares en el debate escuela-trabajo han realizado sugerencias en el sentido de que la capacitación en destrezas laborales y programas de aprendizaje laboral para jóvenes deberían integrarse en la currícula del nivel Polimodal de educación (Hamilton, 1990; Olson, 1993), suministrando a los estudiantes exposición real al lugar de trabajo antes de su graduación.

Aunque estas sugerencias poseen un atractivo inicial, generalmente no han sido uniformemente aceptadas o implementadas (Olson, 1997a), y creemos que la mayoría de las propuestas actuales representan sólo una respuesta parcial a las necesidades de desarrollo de carrera de los estudiantes. Desde un punto de vista conceptual y empírico, las iniciativas y programas escuela-trabajo frecuentemente se encuentran divorciadas de la extensa teoría e investigación básica sobre desarrollo de carrera (Blustein, 1991; Worthington \& Juntunen, 1997), un marco que podría otorgar mayor coherencia y alcance a los esfuerzos actuales. En verdad, el conocimiento de problemáticas tales como las tareas y necesidades de desarrollo de los estudiantes de edades variadas, las variables intervinientes cognitivas a través de las cuales operan los efectos de las intervenciones o los factores socio-contextuales (apoyo familiar y mensajes de los pares, por ejemplo) que facilitan u obstaculizan el desarrollo de carrera, serían beneficiosas para los esfuerzos de intervención. No obstante, además de la disponibilidad de programas comprensivos de orientación y del compromiso de algunos expertos en carreras para diseñar políticas escuela-trabajo, la literatura de desarrollo de carrera ha sido sorprendentemente poco empleada como fundamento (base o suministro) para la investigación y la práctica de la transición al trabajo (Gysbers, 1997).

\section{Un enfoque SCCT de la transición de la escuela al trabajo}

Las nuevas iniciativas escuela-trabajo suministran un particular desafío para los orientadores quienes deben diseñar puentes más efectivos hacia la inserción en el trabajo, considerando los procesos que facilitarán el desarrollo y adaptación a largo plazo de los estudiantes. Más que entender la transición al trabajo como un evento aislado, circunscrito al período inmediatamente precedente y posterior a la graduación de la escuela media, la teoría SCCT entiende esta transición como un proceso que ocurre gradualmente durante los años escolares y posteriormente. Una perspectiva de 
"proceso" versus de "evento", ampliamente aceptada por autores reconocidos de la psicología vocacional (e.g., Super, 1980), entiende la transición hacia el trabajo como un elemento dentro de la estructura mayor del proceso de desarrollo de carrera, donde el ingreso al mundo del trabajo es precedido por un extenso período de preparación y seguido por un igualmente extenso período de ajuste al trabajo y/ o otros roles de la vida.

Debido a que la transición exitosa hacia el trabajo depende del recorrido de los estudiantes por muchas tareas iniciales de desarrollo de carrera (cristalización de los intereses, exploración de carreras, formación en habilidades de toma de decisión, por ejemplo), los intentos para comprender y facilitar esta transición podrían perfectamente comenzar en la escuela elemental y media, continuando hasta (y después) el ingreso de los estudiantes al trabajo (Blustein, Phillips, Jobin-Davis, Finkelberg, \& Roarke, 1997; Fouad, 1997; Worthington \& Juntunen, 1997). Los creadores de programas comprensivos de orientación actualmente en vigencia (Gysbers, 1997) y del movimiento de educación para la carrera (Hoyt, 1977; Isaacson \& Brown, 1993) sostienen posturas semejantes. Por consiguiente, intervenciones y estudios evolutivamente apropiados, deberían ser distribuidos durante los años de escolaridad, en lugar de concentrarse solamente en los estudiantes de Polimodal y la adquisición de destrezas laborales. Adicionalmente, esos programas de investigación e intervención podrían ser mejorados por modelos teóricos que incluyan una perspectiva amplia del fenómeno del desarrollo de carrera, desde la niñez a la adultez.

\section{Procesos y Constructos básicos SCCT}

Nosotros proponemos que SCCT es un modelo heurístico que permite conceptualizar el proceso escuela-trabajo (STW, por sus siglas en ingles, school-to-work), y que puede ser particularmente útil para diseñar intervenciones STW (Lent, Hackett, \& Brown, 1996). En esta sección, primero delinearemos brevemente algunos de los principales conceptos SCCT, para examinar posteriormente como la teoría puede ayudar a comprender y facilitar el proceso STW.

La teoría Social cognitiva enfatiza el interjuego entre tres variables claves, autoeficacia, expectativas de resultados y metas (Bandura, 1986). La autoeficacia, que se refiere a la confianza que poseen las personas en sus habilidades para realizar 
exitosamente una tarea o grupo de tareas determinadas, es percibida como una variable que ayuda a explicar si un individuo tendrá iniciativa, perseverará y tendrá éxito en un determinado curso de acción. Las creencias de autoeficacia provienen de cuatro fuentes primarias de información de eficacia, en interacción con "imputs personales," tales como género, raza/etnicidad, estatus de salud/discapacidades y otras características personales y predisposiciones genéticas (Lent et al., 1994). La fuente más importante de eficacia es la experiencia personal de logros de ejecución: un rendimiento exitoso incrementa la autoeficacia, la que a su vez, aumenta las probabilidades de rendimiento efectivo futuro; las experiencias de fracaso tienden a disminuir la autoeficacia y el rendimiento posterior. Además de las experiencias de logro, la autoefícacia es influida por el aprendizaje vicario u observacional, la persuasión social (aliento o desaliento verbal, por ejemplo); y las reacciones fisiológicas y emocionales (ansiedad debilitante, estados de ánimo positivos, por ejemplo) (Bandura, 1986).

Mientras que la autoeficacia se refiere a las expectativas respecto a nuestras propias capacidades, las expectativas de resultados aluden a las creencias acerca de que ocurrirá como contingente al rendimiento. Vale decir, las expectativas de resultados conciernen a las consecuencias (resultados) de los esfuerzos comportamentales (Bandura, 1986). Los dos tipos de creencias son importantes para orientar la conducta. Así, por ejemplo, un individuo puede pensar que podría completar exitosamente su educación secundaria, pero esas creencias de autoeficacia pueden no ser suficientes para sostener los esfuerzos académicos si la persona perciben que no obtendrá resultados positivos (internos o externos) como producto de esos esfuerzos. Las metas, el tercer elemento básico en el modelo SCCT, se relacionan con la determinación personal para comprometerse en una actividad determinada o para alcanzar un resultado futuro, tales como finalizar una materia difícil de la escuela secundaria, graduarse, ingresar en una pasantía u obtener un empleo determinado. Las metas constituyen un mecanismo crítico mediante el cual las personas ejercen agencia o control personal (Lent et al., 1994). Esto es, mediante su selección de metas, las personas orientan sus propios comportamientos académicos o vocacionales. 
La figura 1 suministra una versión simplificada de las interrelaciones entre estos constructos claves SCCT, y particularmente de como los mismos subyacen al proceso de transición entre la escuela y el trabajo. Aunque en la Figura 1 las vías causales fluyen en una sola dirección, los procesos social cognitivos en realidad son percibidos como recíprocos y continuos. (Para una descripción detallada de las hipótesis SCCT y su estatus empírico, véase Lent et al., 1994; y Lent, Brown, \& Hackett, 1996.)

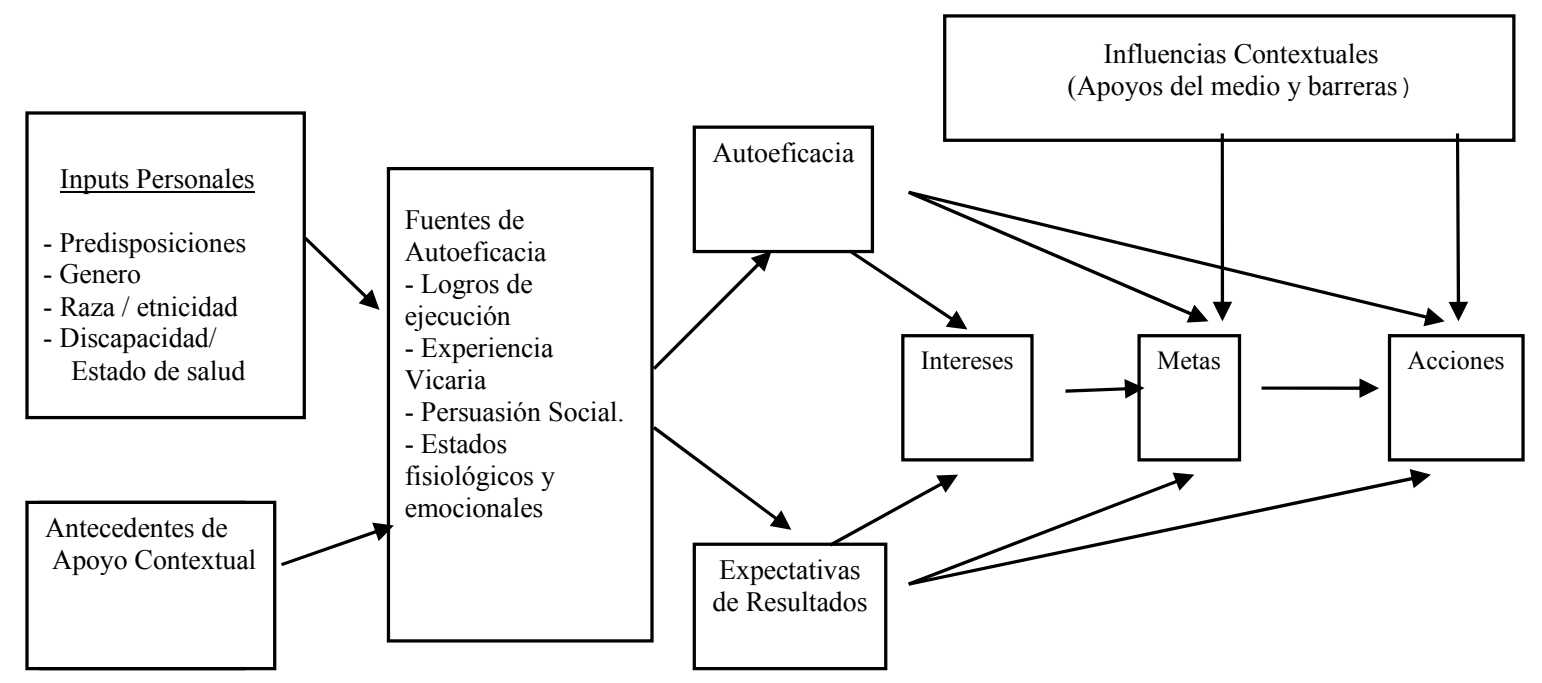

Una perspectiva SCCT de la transición al trabajo per se permitiría esclarecer un conjunto de procesos interrelacionados que se manifiestan en varios momentos del desarrollo, incluyendo: (a) adquisición de autoeficacia y expectativas de resultados realistas y positivas; (b) desarrollo de intereses académicos y de carrera; (c) vínculos entre intereses y metas relacionadas con carreras; (d) traducción de las metas en acciones concretas; (e) facilitación de destrezas académicas y laborales, así como comprender las intervenciones diseñadas para resolver problemas relacionados con el rendimiento; y (f) negociación de apoyos y obstáculos sociales que puedan afectar el desarrollo de creencias ocupacionales y de sí mismo, así como la prosecución de las opciones académicas o de carrera preferidas. Mientras que estos seis procesos son vistos como iterativos y recurrentes, algunos de ellos son especialmente relevantes durante los años de la escuela elemental y media básica, y otros representan las experiencias de los estudiantes en la escuela media especializada (Polimodal), frente a la inminente perspectiva del ingreso al mundo ocupacional. De manera consistente con 
esta perspectiva de la transición hacia el trabajo como resultado de un proceso que se manifiesta durante los años escolares, examinaremos a continuación como se revelan estos procesos en diferentes estadios del desarrollo.

\section{Procesos Sociocognitivos de carrera en la escuela primaria y media básica (EGB)}

De acuerdo con la teoría SCC, los tres primeros procesos que hemos mencionado adquisición de autoeficacia y expectativas de resultados, desarrollo de intereses significativos de carrera y formación de metas y aspiraciones de carrera provisorias representan tareas prominentes durante la escuela media básica y primaria, aunque todas ellas son continuamente revisadas y refinadas durante los años de Polimodal y posteriormente. En los primeros años, los niños habitualmente no poseen un conocimiento adecuado de sus capacidades ni de muchas opciones de carrera, y, por su experiencia limitada, sus intereses y metas vocacionales son bastante estereotipados e inestables. No obstante, al incrementarse su experiencia con una variedad de tareas y comenzar a desarrollar conceptos diferenciados de su eficacia en diversas actividades así como de los resultados de diferentes cursos de vida - sus intereses se transforman en más definidos y estables. Estos intereses emergentes, en combinación con la autoeficacia y las expectativas de resultados, orientarán entonces la formulación y periódica revisión de las metas relevantes para las carreras.

La secuencia hipotetizada sería aproximadamente como la siguiente: Los niños y adolescentes concluyen que "Yo soy bueno en esta actividad (fútbol, pintura, matemática, por ejemplo) y disfruto de las cosas que obtengo practicándola (sentido de satisfacción, buenas notas, aprobación familiar, tiempo libre con mis amigos)". Estas percepciones de competencia y expectativas hacia las futuras recompensas, intrínsecas y extrínsecas, generan intereses en la actividad (por ej., "me gusta tocar el piano") y estimulan las intenciones para continuar comprometiéndose en ella ("Continuaré practicando en casa cuando regrese de la escuela"), así como las fantasías de carrera y metas provisorias ("Cuando sea grande seré un pianista de jazz"). Por el contrario, las creencias de autoeficacia ("Soy malo para las matemáticas") y expectativas de resultados negativas ("Si los demás me ven como demasiado dedicado a las matemáticas, no seré popular") provocan disgusto y evitación de esas actividades particulares, excluyéndolas del rango percibido de opciones de carrera del niño. 
Obviamente, las experiencias de rendimiento son continuas y variables, suministrando amplias oportunidades para revisar las percepciones de sí mismo y de los resultados (por ej., "no estoy seguro de rendir bien en matemáticas durante este trimestre) y, a su vez, para modificar los intereses y metas. Además de interpretar sus propias experiencias de logro, los niños realizan inferencias acerca de su eficacia y los resultados probables que alcanzarán en diferentes actividades mediante el apoyo o desaliento verbal que reciben, la observación de los comportamientos vocacionales y académicos de otras personas y de sus propios estados físicos y reacciones emocionales. Estas diversas fuentes de eficacia e información de rendimiento, distribuidas en las múltiples actividades a las cuales los niños y adolescentes son expuestos vicariamente, ayudan a explicar el fluir inevitable de las metas e intereses de carrera de los más jóvenes.

Creencias de autoeficacia y resultados. El análisis precedente, así como las investigaciones realizadas sobre las hipótesis básicas SCCT, sugieren que las expectativas de resultados y la autoeficacia son centrales para el desarrollo de los intereses académicos y vocacionales de los estudiantes y el rango percibido de opciones ocupacionales (Lent et al., 1994; Lent, Brown, \& Hackett, 1996). Una consecuencia importante de lo anteriormente expresado es que las posibilidades de desarrollo de carrera de los estudiantes pueden ser obstaculizadas a una edad temprana si sus ambientes les ofrecen una exposición sesgada o limitada a experiencias particulares de construcción de la efícacia (en actividades relacionadas con la matemática, por ejemplo) o si adquieren expectativas imprecisas de autoeficacia o resultados. Por consiguiente, los intentos evolutivamente orientados para promover creencias favorables de autoeficacia y expectativas de resultados y para optimizar destrezas básicas, son probablemente más exitosos durante los años de EGB, antes que los intereses y opciones de carrera percibidas por los estudiantes hayan cristalizado totalmente.

Las fuentes de información sobre la eficacia, mencionadas previamente, suministran una estructura para organizar intervenciones psicoeducacionales basadas en la eficacia. Por ejemplo, desde el momento que se asume que los logros de rendimiento personal constituyen una fuente especialmente potente de eficacia percibida, las intervenciones tendientes a incrementar la autoeficacia bien podrían enfatizar las 
experiencias de maestría, facilitando el dominio de contenidos académicos relevantes por parte de los estudiantes. En función de sus vinculaciones recíprocas, las creencias de autoeficacia deberían ser consideradas al unísono con las destrezas objetivas: la autoeficacia realista y fuerte proviene de experiencias de maestría con una tarea particular o una disciplina y, a su vez, contribuye al rendimiento exitoso subsecuente y al desarrollo de destrezas dentro de esos dominios.

La teoría Social cognitiva sugiere que las experiencias de maestría por sí mismas no permiten instaurar creencias robustas de autoeficacia. Se debería prestar atención a los procesos cognitivos, o filtros, a través de los cuales los estudiantes interpretan la calidad de sus logros. El desempeño en matemática ofrece un buen ejemplo. Algunas estudiantes de EGB se desempeñan bastante bien en sus cursos de matemática pero desmerecen sus logros concluyendo, por ejemplo, que "el examen fue fácil" o que "tuve suerte para sacarme una buena calificación" La literatura sugiere que las niñas frecuentemente atribuyen sus logros en matemática, ciencias y otras actividades no tradicionales para mujeres a factores distintos de sus habilidades (Hackett, 1995). Lamentablemente estos patrones atribucionales, con frecuencia frecuentemente aprendidos vicariamente, pueden debilitar la eficacia aun frente al hecho objetivo de las experiencias exitosas. Los intentos para modificar la autoeficacia o las habilidades necesitan, además, incluir procedimientos de reestructuración cognitiva tendientes a promover atribuciones de rendimiento funcionales para la autorrealización.

Las restantes tres fuentes de eficacia personal también pueden suministrar elementos valiosos para la intervención, aumentando o modificando potencialmente los efectos de las experiencias directas de rendimiento. Así, por ejemplo, las estrategias de modelado pueden ser empleadas para ayudar a ciertos estudiantes (mujeres, personas de color, por ejemplo) a considerar áreas de carreras que previamente no habían tenido en cuenta o no habían sido alentados a considerar. Las intervenciones que incorporan persuasión verbal también brindan un medio práctico para fortalecer las destrezas personales y creencias de eficacia. Los mensajes de apoyo pueden asistir a los estudiantes para intentar nuevos comportamientos, persistir pese a fracasos iniciales y a interpretar favorablemente la calidad de sus desempeños. La teoría Social cognitiva también sugiere la importancia de atender a los estados emocionales y fisiológicos, tales 
como ansiedad o afecto negativo, que pueden debilitar la autoeficacia y, además, obstaculizar el rendimiento de los estudiantes.

Los intentos para fortalecer las percepciones de eficacia de niños y adolescentes en un amplio rango de actividades relevantes para las carreras y la escuela pueden ser realizadas por los padres, educadores $u$ orientadores, trabajando juntos o de manera aislada. Sin embargo, los esfuerzos sistemáticos y coordinados parecen tener más probabilidades de ser exitosos, particularmente cuando son incluidos en la currícula escolar y apoyados por iniciativas de la comunidad y el gobierno. Las creencias de eficacia relacionadas con materias específicas (por ejemplo, en relación a las capacidades matemáticas) no deben ser el único aspecto de tales esfuerzos. También parece ser importante desarrollar múltiples facetas de la autoeficacia y la competencia en dominios de habilidades más genéricos -- tales como trabajo en equipo, negociación, liderazgo e interacciones multiculturales - que son percibidas como parte integrante de una transición exitosa al trabajo (Secretary's Commission on Achieving Necessary Skills, 1991).

Intereses y relaciones entre metas e intereses. En vista del hipotetizado impacto causal sobre los intereses y las metas, hemos subrayado intervenciones para promover creencias de autoeficacia y resultados realistas y positivas en los estudiantes de EGB. Además de este énfasis en fertilizar el suelo desde el cual las aspiraciones de carrera pueden surgir, SCCT sugiere un cuidado directo de los intereses y metas en ciernes de los estudiantes. Así, por ejemplo, intervenciones apropiadas a la edad pueden ayudar a los estudiantes en la exploración de sus intereses y las carreras que puedan ser compatibles con ellos. Las destrezas en toma de decisiones y establecimiento de metas también pueden ser fortalecidos, utilizando ejemplos en dominios tales como estudiar o desarrollar amistades, puesto que ambos son significativos para las personas jóvenes y pueden ser fácilmente transferidas a las tareas de desarrollo de carrera.

Mientras que algunas de las sugerencias anteriores también pueden derivarse de otras teorías de carrera, la perspectiva social cognitiva enfatiza que las destrezas, valores, metas, intereses y otras actividades de evaluación deben abordarse muy tentativamente, dado el estatus naciente de estos atributos en edades tempranas. Por consiguiente, los estudiantes, sus padres y maestros deberían ser informados, por ejemplo, acerca que las destrezas e intereses no son estáticos ni inmutables; y que 
pueden modificarse y desarrollarse como función de las experiencias de los niños tanto en la escuela como fuera de ella. Este mensaje puede contradecir las concepciones populares sobre estos atributos (tales como, "Todo es innato;" "la elección de carrera es un simple asunto de aparear rasgos fijos con cualidades de trabajo fijas"), alentando, en realidad, un sentido de agencia personal y colectiva con respecto al desarrollo de carrera de los estudiantes.

Una perspectiva social cognitiva también debería reconocer que muchas personas basan sus decisiones de carrera en factores distintos que sus intereses. Además de la utilidad global de los intereses en la predicción de la elección (Betsworth \& Fouad, 1997), hay necesidad de considerar factores que moderan (debilitan o fortalecen) las relaciones entre intereses y elecciones o que simplemente afectan a los intereses en su impacto sobre la elección. Así, por ejemplo, factores sociales, físicos, educativos y financieros pueden determinar que la opción preferida por un individuo se cambie por otra más fácilmente alcanzable o aceptable para su sistema de apoyo. Bajo tales condiciones, la elección ocupacional puede ser guiada por la accesibilidad de las opciones actuales (que trabajo está disponible?), la autoeficacia (puedo hacer yo ese trabajo?), y las expectativas de resultados (que beneficios obtendré del mismo? que ocurrirá si no lo emprendo?) más que por los intereses personales. Del mismo modo, SCCT postula influencias directas desde la autoeficacia y las expectativas de resultados hacia los comportamientos de elección, además de sus efectos indirectos a través de los intereses (ver Figura 1).

Tal como expresa Bandura (comunicación personal, Marzo 1, 1993), "las personas no eligen trabajar en líneas de ensamblaje o en minas de carbón ... debido a que tengan intereses en esas ocupaciones." Por las mismas razones, muchas personas que anhelan condiciones socioeconómicas envidiables, modifican voluntariamente sus intereses en favor de otros factores, aceptando resultados extrínsecos deseables (salarios altos, estabilidad laboral, por ejemplo) y trabajando en ocupaciones hacia los cuales ellas sienten indiferencia. Los modelos de elección ocupacional que otorgan prioridad a los intereses necesitan ser enriquecidos por otros modelos que consideren el impacto que las estructuras de oportunidades y otros factores pueden ejercer sobre la elección, además de la influencia de los intereses. 


\section{Rediseñando normas para actividades de exploración de carreras.}

Finalmente, la teoría social cognitiva y su investigación básica puede ser usada como guía para reestructurar las actividades de carrera suministradas habitualmente. A continuación ofrecemos dos ejemplos.

En primer lugar las actividades de "día de carreras" y panelistas invitados, que son habituales en muchas escuelas, son ofrecidas con la intención de exponer a los estudiantes a diferentes opciones de carrera o para motivar el rendimiento escolar vinculándolo con resultados extrínsecos. Sin embargo, esas actividades frecuentemente son desarrolladas prestando poca atención a los factores que pueden promover el aprendizaje vicario. Seleccionar modelos creíbles de "afrontamiento" en lugar de modelos de "maestría", con los cuales los estudiantes puedan identificarse en dimensiones múltiples y significativas (raza, edad, género, clase social, competencia percibida, por ejemplo), puede fortalecer el efecto del modelado. Muchos estudiantes de una escuela media de una ciudad económicamente deprimida, por ejemplo, podrían sentirse menos inspirados por el ejemplo de (a) un abogado de mediana edad, adinerado, aunque semejante en raza, que por (b) un estudiante de Polimodal o Universidad de su barrio cuyos progresos educativos han sido el resultado de perseverar y luchar con los mismos factores que esos estudiantes enfrentan cotidianamente.

Por otro lado, se pueden realizar esfuerzos para que, en el proceso de exploración de carreras y adquisición de información de los estudiantes, se contemplen actividades que faciliten percepciones de eficacia y expectativas de resultados precisas. Así, por ejemplo, los orientadores pueden favorecer el debate acerca de como los estudiantes perciben los requerimientos en destrezas de trabajo en relación a sus habilidades actuales o potenciales. Esos debates pueden revelar que un estudiante está sobreestimando algún requerimiento de habilidad ocupacional ("y o necesito ser un genio para ingresar en este campo laboral", por ejemplo), subestimando sus capacidades, o apreciando equivocadamente su potencial para desarrollar ciertas destrezas con su posterior educación y esfuerzo. La Orientación puede, de este modo, concentrarse en objetivos tales como interpretar la información ocupacional más precisamente, confrontar las autoevaluaciones que parecen inconsistentes con los datos de tests de aptitudes o el rendimiento, y estimular a los estudiantes para que perciban 
sus habilidades personales como un conjunto de destrezas adquiribles más que como una capacidad innata e inmutable (Bandura, 1993).

\section{Facilitadores sociocognitivos de la transición hacia el trabajo en la escuela Polimodal y después del egreso}

Como hemos sugerido anteriormente, el desarrollo de expectativas de resultado y autoeficacia fuertes y realistas funciona como un importante plataforma para la transición exitosa hacia el trabajo, capacitando a los estudiantes para identificar intereses y alternativas de elección potencialmente satisfactorias, y evitando en lo posible las distorsiones cognitivas y las experiencias limitadas. De este modo, en un escenario ideal, los estudiantes han tenido amplias experiencias con una variedad de actividades significativas en relación al trabajo, desarrollando patrones diferenciados de intereses ocupacionales y metas congruentes con sus intereses al final del Polimodal. El modelo de elección SCCT asume que el éxito del proceso de transición al trabajo y de la toma de decisión en este estadio depende ulteriormente de, al menos, tres tareas de desarrollo adicionales, a saber: (a) la habilidad de los estudiantes para traducir sus metas en acciones, (b) sus destrezas de rendimiento en un dominio específico y sus "destrezas generales de empleo" (Worthington \& Juntunen, 1997), y (c) los apoyos y obstáculos previstos y encontrados en la prosecución de sus metas -y como los estudiantes negocian con esos factores.

Relaciones entre metas y acciones. La teoría Social cognitiva otorga un lugar de privilegio a las metas personales, visualizándolas como motivadores claves del comportamiento. Esta teoría identifica también algunas propiedades de las metas que afectan sus probabilidades de ser implementadas. Así, por ejemplo, las metas funcionarán como una guía más confiable del comportamiento en la medida que sean claras, específicas, divididas en sub-metas, temporalmente próximas a las acciones intentadas, establecidas públicamente, y mantenidas con un fuerte compromiso (por el contrario a las metas vagas, amorfas, distantes, y formuladas de manera privada o con compromiso débil) (Bandura, 1986). Los esfuerzos para impartir destrezas efectivas de establecimiento de metas - y particularmente para ayudar a los estudiantes a estructurar sus metas en términos optimamente facilitativos(claridad y especificidad, por ejemplo) - deberían, además, ser claramente garantizados desde una perspectiva SCCT. 
También debería considerarse que el establecimiento de metas ocurre dentro de un contexto social, cultural y económico que puede tener una substancial vinculación con la naturaleza de las metas individuales, así como con el modo en que las personas persiguen o revisan sus metas personales. Un estudio cualitativo reciente ha esclarecido el impacto percibido de los recursos y obstáculos ambientales sobre los resultados del proceso de transición al trabajo de los estudiantes (Blustein et al., 1997). SCCT postula que la habilidad y voluntad de los estudiantes para traducir sus intereses en metas y sus metas en acciones (ingresando en un programa de entrenamiento laboral o postulándose para un empleo, por ejemplo), serán afectadas por sus percepciones de los apoyos ambientales (satisfacción de otras personas significativas con nuestras metas, por ejemplo) y obstáculos (falta de medios económicos para la capacitación, por ejemplo).

Apoyos y obstáculos ambientales. Dados los efectos hipotetizados del ambiente percibido (y real), las intervenciones derivadas de un enfoque SCCT deben considerar los apoyos y obstáculos que los estudiantes piensan que afectarían su transición hacia el trabajo. Así por ejemplo, cuando se emplean estrategias de afrontamiento de obstáculos similares a las descritas por Brown y Lent (1996), los estudiantes pueden ser alentados a examinar los obstáculos potenciales para proseguir sus metas de carrera preferidas, a analizar la probabilidad de su ocurrencia, y a desarrollar estrategias para prevenir y manejar las barreras que ellos probablemente deberán enfrentar. Ayudar a los estudiantes a prever y prepararse para probables obstáculos, tales como desaprobación de los pares o problemas económicas, puede disminuir las oportunidades de que sean sorprendidos y conducidos por las circunstancias desfavorables.

Además de las estrategias de afrontamiento de obstáculos, el modelo SCCT debe prestar consideración al aprovechamiento de los apoyos ambientales y la estructura de oportunidades. Así como los estudiantes pueden ser asistidos para enfrentar obstáculos probables, también pueden ser ayudados a reconocer y acceder a los recursos del entorno que puedan sostener su transición al trabajo. Así, por ejemplo, los estudiantes pueden ser alentados a identificar y utilizar fuentes de búsqueda de trabajo dentro de sus sistemas naturales de apoyo (miembros de su familia o vecinos, por ejemplo), o a vincularse a nuevos grupos de amigos que probablemente apoyarán más sus metas vocacionales que sus grupos de referencia actuales. Las intervenciones 
psicoeducacionales dirigidas a estos aspectos del proceso de transición al trabajo de construcción de apoyos y superación de obstáculos también deberían concentrarse en el sentido de eficacia y afrontamiento de los estudiantes con obstáculos ambientales como el racismo y el sexismo. (Véase Hackett \& Byars, 1996, para una detallada discusión de la eficacia de afrontamiento)

Comportamientos de logro. La elección vocacional, por supuesto, no es una empresa unilateral: mientras las personas seleccionan carreras, también son seleccionadas por ellas, sobre la base de destrezas demostradas y otras consideraciones. Por consiguiente, la prosecución exitosa de una meta personal $-\mathrm{y}$, por añadidura, la estabilidad de la elección y la transición al trabajo - dependen también del rendimiento adecuado del individuo en la escuela y el trabajo.

La hipótesis básica del modelo de rendimiento SCCT postula que las creencias positivas de autoeficacia facilitan el logro en un dominio de desempeño determinado, en la medida que el individuo posea, al menos, niveles mínimamente adecuados de las destrezas requeridas en ese dominio. En términos prácticos, esta hipótesis sugiere que la estimación de autoeficacia puede ser comparada con la información de evaluación objetiva de de destrezas o del rendimiento pasado cuando se intentan identificar las razones del rendimiento pobre de las personas, y para orientar las intervenciones sobre problemas de rendimiento académico o relacionado con el trabajo.

La teoría SCCT predice resultados diferentes según la autoeficacia sea comparable con las destrezas personales actuales. Las creencias de autoeficacia que exceden ligeramente nuestro ( $\mathrm{y}$ al menos adecuado) nivel de destreza actual, son motivadoras del compromiso en las tareas y el desarrollo posterior de habilidades, mientras que las estimaciones globales o la subestimación de autoeficacia probablemente serán más problemáticas (Bandura, 1986). Las evaluaciones excesivamente optimistas también pueden conducir al fracaso y el desaliento, mientras que las poco realistas y negativas respecto a uno mismo puede provocar evitación de las tareas o un despliegue insuficiente de las destrezas personales. Los estudiantes con creencias débiles de autoeficacia pero con adecuadas destrezas en un contexto de desempeño particular pueden beneficiarse de intervenciones destinadas a promover su eficacia y, posiblemente, de futuras actividades de desarrollo de destrezas, dependiendo de su futuro nivel de habilidad. 
La teoría SCCT no afirma que las creencias de autoeficacia compensarán la carencia de destrezas necesarias o que los meros intentos para incrementar la autoeficacia serán suficientes en condiciones en las cuales las destrezas son inadecuadas. Los estudiantes que exhiben creencias débiles de autoeficacia o destrezas insuficientes pueden beneficiarse de las tentativas para reforzar sus habilidades, o del examen de alternativas ocupacionales o académicas que se correspondan con sus capacidades actuales. Por consiguiente, las intervenciones promotoras de eficacia no deberían ser indicadas para todos los estudiantes que experimentan problemas de rendimiento relacionados con la escuela o el trabajo.

No obstante, la teoría y la investigación sugieren el valor de diseñar actividades que ayuden a los estudiantes para : (a) obtener experiencias de maestría personal con tareas ocupacionales o académicas progresivamente más desafiantes; (b) revisar sus experiencias de éxito en el pasado e (c) interpretar sus éxitos anteriores y actuales de manera que promuevan la competencia percibida, más que disminuirla (cf. Brown \& Lent, 1996; Lent, Brown, \& Hackett, 1996).

Es importante resaltar que estas prescripciones para el mejoramiento de las destrezas y la autoeficacia son semejantes a aquéllas descritas anteriormente en relación a los estudiantes más jóvenes. Si esas estrategias son orientadas por fines de desarrollo o correctivos, pueden apoyarse sobre parecidas fuentes de información de la eficacia (logros de rendimiento personales, influencias vicarias, por ejemplo), realizando las modificaciones necesarias para que las intervenciones sean adecuadas a este nivel de edad. Las intervenciones promotoras de eficacia pueden ser especialmente beneficiosas dentro del contexto de programas de exposición al trabajo en el Polimodal, cuando los estudiantes están aprendiendo nuevas destrezas ocupacionales específicas y refinando destrezas generales, tales como responsabilidad y negociación, que, idealmente, han sido adquiridas antes. Así, por ejemplo, los supervisores de las pasantías pueden ser capacitados en métodos para estructurar las condiciones laborales y el feedback que proporcionen, de manera que permita maximizar el desarrollo de la autoeficacia de los estudiantes.

Otras estrategias dirigidas a facilitar las experiencias de aprendizaje basadas en el trabajo también pueden derivarse del modelo SCTT. Quizá el punto fundamental es que, mientras las propuestas de los programas de preparación para el trabajo parecen 
implicar que la exposición aislada al trabajo resulta útil, las teorías e investigación del desarrollo de carrera pueden emplearse para incrementar el alcance de tales programas, por ejemplo, teniendo en cuenta la congruencia entre la persona y el ambiente o reestructurando el entorno laboral para atender las necesidades de desarrollo de los estudiantes. En esencia una sola medida es insuficiente para todos los casos. De este modo, subrayamos nuevamente el valor potencial de dotar de fundamentos teóricos a las iniciativas actuales de transición hacia el trabajo.

\section{Comentarios finales}

Hemos intentado demostrar como el enfoque SCCT puede ser utilizado como un punto de vista privilegiado para comprender el proceso de transición escuela-trabajo. La explicación SCCT de este proceso se concentra en seis aspectos evolutivamente relacionados que se manifiestan en los años de escolaridad, y no solamente en el período inmediatamente previo al egreso del Polimodal. El acento en estos seis aspectos sugiere finalidades tanto para intervenciones asistenciales como preventivas, destinadas a facilitar el desarrollo de carrera de los estudiantes durante la escuela y después de su ingreso al trabajo. Recientemente han surgido unas pocas intervenciones creativas para estudiantes elementales, basadas en el modelo SCCT, incluyendo intentos para promover autoeficacia y expectativas de resultados positivas en ciencia y matemáticas en niñas (Solberg, Edejer, Ryan, \& Zukerman, 1996) y estudiantes de color (Fouad, 1996), así como para estructurar una red "electrónica" de apoyo a la carrera para estudiantes de minorías raciales vía Internet (M.T. Brown, comunicación personal, Febrero 4, 1995).

Mediante este diseño de procesos de desarrollo de carrera óptimos, no queremos hacer pensar que los estudiantes serán (o deberían ser) "productos terminados" después de completar su Polimodal. Por el contrario, la fluidez y algunas modificaciones en el desarrollo de los intereses y la autoeficacia y otros aspectos claves son posibles y aún deseables en el nivel Polimodal (y después), en tanto los estudiantes (y los trabajadores) continúan descubriendo nuevos datos en relación a sus capacidades y posibilidades de carrera.

En realidad, además de las preocupaciones de los políticos acerca de los jóvenes indecisos y su anhelo de crear un sistema de transición al trabajo eficiente y seguro, el 
ajuste a largo plazo de las personas respecto a sus carreras sería puesto en riesgo si sus aspiraciones y atributos de carrera fueran irrevocablemente fijados en la adolescencia o vinculados a la suerte de una industria en particular.

Ciertamente, el mercado laboral no es estático, como evidencian los recientes y vertiginosos avances tecnológicos. En esos contextos dinámicos, los trabajadores son estimulados para el continuo crecimiento y renovación de sus carreras (Watts, 1996). Por consiguiente, las intervenciones de transición pueden transmitir el valor del desarrollo de carrera a lo largo de la vida (explorando regularmente los intereses en nuevas áreas, actualizando las destrezas y aprendiendo a manejar las transiciones, por ejemplo), más que asumir que el desarrollo de carrera finaliza con la graduación del Polimodal y la entrada a la Universidad o el lugar de trabajo. En ese sentido, los cambios de carrera pueden ser abordados como cambios normales en la vida del individuo y no como cambios patológicos, aunque algunas veces hay una línea delgada entre experimentación e indecisión, y entre cambios normales e inestabilidad laboral.

El proceso de transición al trabajo representa un dominio emergente para ser explorado por los orientadores vocacionales o escolares. Con el fin de prevenir contra argumentaciones uniformes y para apreciar la variación inherente a la negociación de este proceso por parte de los estudiantes, tendrá importancia estudiar las experiencias de transición al trabajo en grupos diversos, tales como estudiantes que están en situación de riesgo académico, que provienen de medios económicamente desfavorecidos, miembros de grupos raciales minoritarios, o con discapacidades (Worthington \& Juntunen, 1997).

Esas investigaciones también deberían explorar las diferencias y resultados del proceso de transición dentro de un mismo grupo. También resultará muy valioso dedicar especial consideración a los recursos y los obstáculos, tanto personales como ambientales, que afectan la transición al trabajo de los estudiantes (Blustein et al., 1997). Así, por ejemplo, las influencias negativas de los pares pueden perjudicar cualquier intento bien intencionado para promover motivación académica (Steinberg, Dornbusch, \& Brown, 1992). Un análisis de los modos con que los individuos afrontan esas influencias (incluyendo a aquellos individuos que parecen ser "resilientes" para enfrentar múltiples obstáculos) podría ser empleado para desarrollar estrategias proactivas de afrontamiento de obstáculos. 
Quisiéramos concluir con una nota ecuménica: la transición STW, y otros momentos de transición (jubilación, cambio de carrera o reingreso a la carrera luego del período de crianza de los niños, por ejemplo), podría comprenderse mejor y ser facilitada si se aplicasen una variedad de perspectivas teóricas relacionadas no solo al ámbito de las carreras sino también a otros ámbitos (afrontamiento del estrés, transiciones en la vida, por ejemplo), más que apoyándose en conjeturas ateóricas o burocráticas aisladas. Pese a que hemos acentuado el importante potencial del modelo SCCT para comprender el ajuste al proceso STW, este problema merece mayor atención de teóricos, investigadores y profesionales prácticos, trabajando cooperativamente y a través de los límites de las disciplinas. Estos esfuerzos pueden ser ayudados si se emplease un marco conceptual común y se promoviese la discusión entre varios

profesionales que están preocupados con el progreso educacional de los estudiantes y las transformaciones del mundo del trabajo. Nosotros esperamos que la teoría social cognitiva podrá evidenciar su utilidad si es empleada como uno de esos marcos conceptuales integradores.

\section{Referencias}

Bandura, A. (1986). Social foundations of thought and action: A social cognitive theory. Englewood Cliffs, NJ: Prentice-Hall.

Bandura, A. (1993). Perceived self-efficacy in cognitive development and functioning. Educational Psychologist, 28, 117-148.

Betsworth, D.G., \& Fouad, N.A. (1997). Vocational interests: A look at the past 70 years and a glance at the future. The Career Development Quarterly, 46, 23-47.

Betz, N.E., \& Hackett, G. (1997). Applications of self-efficacy theory to the career assessment of women. Journal of Career Assessment, 5, 383-402.

Blustein, D.L. (1991). On facilitating the transition to adulthood: Are apprenticeships the answer? Contemporary Psychology, 36, 687-688.

Blustein, D.L., Phillips, S.D., Jobin-Davis, K., Finkelberg, S.L., \& Roarke, A.E. (1997). A theory-building investigation of the school-to-work transition. The Counseling Psychologist, 25, 364-402.

Brown, S.D., \& Lent, R.W. (1996). A social cognitive framework for career choice counseling. The Career Development Quarterly, 44, 354-366. 
Chartrand, J.M., \& Rose, M.L. (1996). Career interventions for at-risk populations: Incorporating social cognitive influences. The Career Development Quarterly, 44, 341-353.

Fouad, N.A. (1996, November). Career interventions with middle school youth. Presentation at the Conference on School-to-Work Transition: Defining the Role of Vocational Psychology, Washington, DC.

Fouad, N.A. (1997). School-to-work transition: Voice from an implementer. The Counseling Psychologist, 25, 403-412.

Fouad, N.A., \& Smith, P.L. (1996). A test of a social cognitive modelfor middle school students: Math and science. Journal of Counseling Psychology, 43, 338346.

Gainor, K.A., \& Lent, R.W. (in press). Social cognitive expectations and racial identity attitudes in predicting the math enrollment intentions of Black college students. Journal of Counseling Psychology.

Gysbers, N.C. (1997). Involving counseling psychology in the school-to-work movement: An idea whose time has come. The Counseling Psychologist, 25, 413-427.

Hackett, G. (1995). Self-efficacy and career choice and development. In A. Bandura (Ed.), Self-efficacy in changing societies (pp. 232-258). New York: Cambridge University Press.

Hackett, G., \& Byars, A. M. (1996). Social cognitive theory and the career development of African American women. The Career Development Quarterly, 44, 322-340.

Hamilton, S.F. (1990). Apprenticeship for adulthood: Preparing youth for the future. New York: Free Press.

Hoyt, K. B. (1977). A primer for career education. Washington, D.C.: Office of Career Education.

Isaacson, L. E., \& Brown, D. (1993). Career information, career counseling, and career development (5th ed.). Boston: Allyn \& Bacon.

Lapan, R.T., Shaughnessy, P., \& Boggs, K. (1996). Efficacy expectations and vocational interests as mediators between sex and choice of math/science college majors: A longitudinal study. Journal of Vocational Behavior, 49, 277-291. 
Lent, R. W., Brown, S. D., \& Hackett, G. (1994). Toward a unifying social cognitive theory of career and academic interest, choice, and performance [Monograph]. Journal of Vocational Behavior, 45, 79-122.

Lent, R. W., Brown, S. D., \& Hackett, G. (1996). Career development from a social cognitive perspective. In D. Brown, L. Brooks, \& Associates, Career choice and development: Applying contemporary theories to practice (3rd ed., pp. 373-421). San Francisco: Jossey-Bass.

Lent, R.W., Hackett, G., \& Brown, S.D. (1996). A social cognitive framework for studying career choice and transition to work. Journal of Vocational Education Research, 21(4), 3-31.

Lopez, F.G., Lent, R.W., Brown, S.D., \& Gore, P.A. (1997). Role of social-cognitive expectations in high school students' mathematics-related interest and performance. Journal of Counseling Psychology, 44, 44-52.

Olson, L. (1993, June 23). Clinton to urge state systems for school-job link. Education Week, pp. 1, 36 .

Olson, L. (1997a, May 21). State progress on school-to-work reforms slow, study finds. Education Week, p. 18.

Olson, L. (1997b). The school-to-work revolution. Reading, MA: Addison-Wesley.

Secretary's Commission on Achieving Necessary Skills (1991). What work requires of schools: A SCANS report for America 2000. Washington, DC: U.S. Department of Labor.

Solberg, V.S., Edejer, E., Ryan, N., \& Zukerman, D. (1996, August). Social cognitive interventions designed to increase representation of women in science. Paper presented at the meeting of the American Psychological Association, Toronto, $\mathrm{ON}$.

Steinberg, L., Dornbusch, S.M., \& Brown, B.B. (1992). Ethnic difference in academic achievement: An ecological perspective. American Psychologist, 47, 723-729.

Super, D. E. (1980). A life-span, life-space approach to career development. Journal of Vocational Behavior, 13, 282-298.

Watts, A.G. (1996). Toward a policy for lifelong career development: A transatlantic perspective. The Career Development Quarterly, 45, 41-53. 
Worthington, R.L., \& Juntunen, C.L. (1997). The vocational development of noncollege-bound youth: Counseling psychology and the school-to-work transition movement. The Counseling Psychologist, 25, 323-363. 